\title{
Changes to aspects of ongoing randomised controlled trials with fixed designs
}

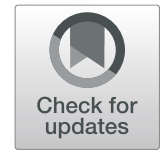

\author{
Xanthi Coskinas ${ }^{1}$, John Simes ${ }^{1}$, Manjula Schou ${ }^{2,3}$ and Andrew James Martin ${ }^{1 *}$ (D)
}

\begin{abstract}
Background: Despite careful planning, changes to some aspects of an ongoing randomised clinical trial (RCT), with a fixed design, may be warranted. We sought to elucidate the distinction between legitimate versus illegitimate changes to serve as a guide for less experienced clinical trialists and other stakeholders.

Methods: Using data from a large trial of statin therapy for secondary prevention, we generated a set of simulated trial datasets under the null hypothesis $(\mathrm{HO})$ and a set under an alternative hypothesis ( $\mathrm{H} 1)$. Through analysis of these simulated trials, we assessed the performance of the strategy of changing aspects of the design/analysis with knowledge of treatment allocation (illegitimate) versus the strategy of making changes without knowledge of treatment allocation (legitimate). Performance was assessed using the type 1 error, as well as measures of absolute and relative bias in the treatment effect.
\end{abstract}

Results: Illegitimate changes led to a relative bias of $61 \%$ under $\mathrm{H} 1$, and a type 1 error rate under $\mathrm{H} 0$ of $23 \%$-well in excess of the $5 \%$ significance level targeted. Legitimate changes produced unbiased estimates under $\mathrm{H} 1$ and did not inflate the type 1 error rate under $\mathrm{HO}$.

Conclusions: Changes to pre-specified aspects of the design and analysis of an ongoing RCT may be a necessary response to unforeseen circumstances. Such changes risk introducing a bias if undertaken with knowledge of treatment allocation. Legitimate changes need to be adequately documented to provide assurance to all stakeholders of their validity.

Keywords: Randomised control trials, Bias, Design changes, Type 1 error

\section{Background}

Despite the emergence of adaptive trial designs such as multi-arm multi-stage (MAMS) designs [1] and adaptive Bayesian designs [2], the standard phase III randomised controlled trial (RCT) framework characterised by the pre-specification of fixed design elements remains the mainstay of clinical research. Despite careful prospective planning, changes to some aspect of an ongoing RCT, with a fixed design, may be warranted in reaction to new

\footnotetext{
* Correspondence: andrew.martin@ctc.usyd.edu.au

${ }^{1}$ The National Health and Medical Research Council Clinical Trial Centre, University of Sydney, Camperdown, NSW 2050, Australia

Full list of author information is available at the end of the article
}

information arising. The new information triggering a reactive revision could potentially come from a variety of sources, including: another recently completed RCT investigating a similar question; basic research revealing new insights into the pathophysiology of a disease; and/ or a clinical study clarifying the pharmacokinetic/pharmacodynamic properties of an experimental treatment. Reactive revisions to the planned analysis approach may arise as a consequence of seeking greater statistical power (to correctly reject the null hypothesis) and/or to accommodate unexpected characteristics of the trial data. Some examples of reactive revisions to the primary endpoint, the analysis set composition and the analysis method are presented in Table 1. 
Even when no reactive revision is made to aspects of an RCT, there may remain opportunities for flexibility in the form of discretionary decisions relating to analysis details that were not specified in the original protocol. Examples could include: whether or not to exclude from the analysis set a small proportion of patients found (after randomisation) to have been ineligible (on the basis of pre-randomisation factors); refinement of an ambiguous endpoint definition [7]; whether or not to adjust for a particular baseline covariate; or the choice of statistical test to compare randomised groups on a given endpoint.

Reactive revisions and discretionary decisions represent mechanisms for changing design and/or analysis aspects of an ongoing RCT, and such changes may bias results. The potential for bias rests on whether the change is based on information that is completely independent of treatment allocation, as opposed to information that is related to treatment allocation. Changes should be regarded as legitimate if the former can be assured and the revision is otherwise sound (i.e. the revised question is still regarded as important, the revised design has adequate power, the study remains ethical, etc.).

The fundamental distinction between legitimate versus illegitimate changes is no doubt well understood by experienced trialists, biostatisticians and clinical epidemiologists. Evidence of illegitimate changes nevertheless arising in the literature [8-16] suggests that there is value in illustrating the distinction to a broader constituency of stakeholders in clinical trials research to help ensure that: less experienced trialists do not inadvertently introduce (the perception of) a bias to their RCT; and reviewers/peers do not incorrectly judge legitimate changes as threats to the credibility of a well-conduced

\section{Table 1 Examples of reactive revisions}

1. A reactive revision to the choice of primary endpoint occurred in the FIELD trial. The FIELD trial randomised 9795 patients with type 2 diabetes mellitus to fenofibrate (a drug that modifies the lipid profile) or placebo [3]. The original primary endpoint of coronary heart disease (CHD) death was changed to 'any coronary event' on the basis of a blinded (pooled) review of interim data that indicated a lower than expected CHD death rate that was inadequate for reaching the statistical power target.

2. A reactive revision to the analysis set composition arose in the $\mathrm{CO} 17$ randomised controlled trial of cetuximab in patients with pre-treated metastatic epidermal growth factor receptor (EGFR)-positive colorectal carcinoma in response to external evidence suggesting that K-ras wildtype was a predictive biomarker. The effect of cetuximab was indeed found to be largely restricted to this subset of randomised patients [4, 5].

3.The Sentinel Node Biopsy versus Axillary Clearance (SNAC1) trial changed the primary analysis from a comparison of proportions (of patients experiencing lymphoedema) to a comparison of means (of arm swelling) to take advantage of the greater statistical power of parametric analysis methods [6].
RCT. We sought to achieve this via a simulation study following the framework of Morris et al. [17].

\section{Aim}

This study aimed to investigate the effect of allowing various changes to the planned design/analysis of an $\mathrm{RCT}$, with and without knowledge of treatment allocation, under a scenario where there is no true difference between treatment groups ( $\mathrm{H} 0$, null hypothesis) and when there is a true difference between treatment groups ( $\mathrm{H} 1$, alternative hypothesis).

\section{Methods}

\section{The Long-term Intervention with Pravastatin in Ischemic Disease (LIPID) trial}

We chose to undertake the simulation study using data from a real RCT, rather than completely simulating the data, because this avoided the need to make assumptions about the joint probability distribution of patient baseline characteristics and outcomes. We used data from the LIPID trial that randomised-to pravastatin (a drug that modifies the lipid profile) or to placebo-a total of 9014 patients who had experienced an acute myocardial infarction (AMI) or a hospital discharge diagnosis of unstable angina pectoris (UAP) in the preceding 3-36 months [18]. Patients were recruited from 87 sites and were followed over a median of 6 years. The primary analysis for LIPID specified: time to CHD death as the primary endpoint; all randomised patients as the analysis set; and no adjustment for any baseline patient characteristics [18]. Cox proportional hazards regression was used to express the treatment effect as a hazard ratio (HR).

\section{Data-generating mechanisms}

We constructed 100,000 simulated LIPID trial datasets under the null hypothesis ( $\mathrm{H} 0)$, where a $\mathrm{HR}$ of 1 was imposed (reflecting no difference in the hazard of CHD death between experimental and control treatment groups), and 100,000 simulated LIPID trial datasets under an alternative hypothesis (H1), where a HR of 0.85 was imposed (i.e. the hazard of CHD death was $15 \%$ lower in the experimental group compared to the control group). Full details of how this was done are presented in Additional file 1. All simulated trials were analysed using Cox proportional hazards regression, where any one of 72 unique analysis configurations could be applied to the analysis of an individual simulated trial. The configurations were constructed via a combination of options presented in Table 2 relating to: the choice of endpoint (three options); the analysis set composition (three options); and covariate specification (eight options). More information on these analysis configurations is presented in Additional file 1. Each configuration 
Table 2 Analysis configurations arising from three decision categories

\begin{tabular}{|c|c|}
\hline Type of decision & Decision options \\
\hline Choice of endpoint & $\begin{array}{l}\text { 1. Coronary heart disease }(\mathrm{CHD}) \text { death }^{\mathrm{a}} \\
\text { 2. Revascularisation } \\
\text { 3. All-cause mortality }\end{array}$ \\
\hline Analysis set composition & $\begin{array}{l}\text { 1. No exclusions (i.e. all randomised patients) } \\
\text { 2. Exclude patients with eGFR < } 45 \text { (Stage } 3 \text { a kidney disease or worse) (excludes 4\%) } \\
\text { 3. Exclude patients with qualifying events within } 9 \text { months of randomisation (excludes 29\%) }\end{array}$ \\
\hline Covariate specification & $\begin{array}{l}\text { 1. None } \\
\text { 2. Stroke ( } 2 \text { levels: yes, no) } \\
\text { 3. Smoking status ( } 2 \text { levels: never smoked, current/ex-smoker) } \\
\text { 4. Index ACS ( } 3 \text { levels: unstable angina pectoris (UAP), single myocardial infarction (MI), multiple MI) } \\
\text { 5. Stroke and smoke } \\
\text { 6. Stroke and index ACS } \\
\text { 7. Smoke and index ACS } \\
\text { 8. Stroke, smoke and index ACS }\end{array}$ \\
\hline
\end{tabular}

ACS acute coronary syndrome, eGFR estimated glomerular filtration rate

aase-case analysis: CHD death, no exclusions, no baseline covariate adjustment

was considered a methodologically reasonable optionwe did not investigate options with a known potential to introduce a bias, such as per-protocol type analyses that exclude patients with poor adherence to protocol therapy.

\section{Targets and analysis methods}

The targets for our simulation studies were the log hazard ratio $(\theta)$ and type I error. We used the coefficient

$(\hat{\theta})$ for treatment obtained from a Cox proportional hazards model as the estimate for the estimand $(\theta)$, and used the Wald test associated with $\hat{\theta}$ to produce a $p$ value.

\section{Performance measures}

We estimated the following:

- the type I error rate using $\frac{1}{n_{\text {sim }}} \sum_{i=1}^{n_{\text {sim }}} 1\left(p_{i} \leq \alpha\right)$, where $p_{i}$ is the $p$-value associated with the null hypothesis

- the expectation of $\hat{\theta}$ as $E[\hat{\theta}]=\frac{1}{n_{\text {sim }}} \sum_{i=1}^{n_{\text {sim }}} \hat{\theta}_{i}$;

- the bias of $\hat{\theta}$ as $\frac{1}{n_{\text {sim }}} \sum_{i=1}^{n_{\text {sim }}} \hat{\theta}_{i}-\theta$; and

- the relative bias of $\hat{\theta}$ as $\frac{\left(\frac{1}{n_{\text {sim }}} \sum_{i=1}^{n_{\text {sim }}} \hat{\theta}_{i}\right)-\theta}{\theta}$.

\section{Analysis selection strategy}

For each simulated trial, two classes of strategy were used to determine whether or not to switch from the base-case analysis (the actual primary analysis specified in the LIPID trial) and select (for reporting) one of the other possible analysis configurations. Strategy A involved switching at random, with $50 \%$ probability, from the base-case analysis to a random choice of one of the other 71 possible alternative configurations. Strategy A consequently represented a legitimate approach to making changes to the original design/planned analysis of a RCT without knowledge of the association between treatment allocation and outcome.

Strategy B involved selecting (to report) the analysis configuration that yielded the most extreme result for a given simulated trial. We operationalised the selection of the 'most extreme result' in two ways. The first, Strategy B1, was to select the analysis with the smallest (twosided) $p$-value reflecting a situation where a 'statistically significant' finding is being inappropriately sought to, for example, misguidedly improve the likelihood of results publication. The direction of the treatment effect should have no bearing on the choice of the analysis configuration under Strategy B1 as a choice based on the smallest $p$-value could favour either treatment. The second way, Strategy B2, was to select the analysis with the smallest $\hat{\theta}$-reflecting a situation where a stronger effect is being inappropriately sought to, for example, improve the estimated incremental effectiveness of a new therapy. Strategies B1 and B2 simulated an approach to making illegitimate changes with knowledge of the association between treatment allocation and outcome.

\section{Scenarios evaluated}

The change strategies were compared under three scenarios. For Scenario 1, simulations were performed under $\mathrm{HO}(\theta=0)$ and all of the 72 possible analysis configurations were available for selection. Strategy A was compared against Strategies B1 and B2.

For Scenario 2, simulations were performed under $\mathrm{HO}$ $(\theta=0)$ but constraints were imposed on the selection of possible analysis configurations. Three types of constraint were investigated. The first allowed flexibility away from the base-case only in the selection of the 
analysis endpoint (three configurations). The second allowed flexibility away from the base-case only in the selection of the analysis set (three configurations). The third allowed flexibility away from the base-case only for covariate specification (eight configurations). The simulations conducted under Scenario 2 thus allowed us to explore the isolated effect of changing only one aspect of the trial while keeping the other aspects fixed.

For Scenario 3, simulations were performed under H1 comparing Strategies A and B. Under H1, a HR of 0.85 was imposed on the CHD death endpoint only (see Additional File 1). For analyses of the simulated data that were restricted to the CHD death endpoint only, $\theta=$ $\log (0.85)=-0.1625$. The effect we imposed on CHD death indirectly introduced a treatment effect on the revascularisation and all-cause mortality endpoints because there was some correlation between these events and CHD. Thus, $\theta=-0.1262$ was determined to be the appropriate estimand for analyses of the simulated data with no restriction on the choice of endpoint.

\section{Results}

\section{Scenario 1}

Under H0, Strategy A produced unbiased results. The $p$ values had a uniform distribution (Fig. 1a), and $\hat{\theta}$ was symmetrically distributed with $E[\hat{\theta}]=0.000$ (Fig. 1b). The type 1 error rate of Strategy A was consistent with the 5\% significance level targeted (mean: 5.0\%, 95\% CI: 4.9\% to $5.2 \%)$. The results for Strategies B1 and B2 were biased under H0. For Strategy B1, p-values were right skewed (Fig. 1a), and the type 1 error rate was well above the 5\% level (mean: 23.0\%, 95\% CI: 22.7 to 23.2). For Strategy B2, $\hat{\theta}$ was symmetrically distributed but biased with $E[\hat{\theta}]=-0.0737$ (Fig. 1b).

\section{Scenario 2}

When flexibility in analysis configuration was permitted in only one of the three analysis decision categories under H0, Strategy A again produced unbiased results (in each case $E[\hat{\theta}]=0.000$ ). The bias introduced under Strategies B1 and B2 was greatest when there was freedom to switch only the endpoint (Fig. 2a1, a2), followed by the analysis set composition (Fig. 2b1, b2) and, finally, the covariate specification (Fig. 2c1, c2).

\section{Scenario 3}

In the initial series run under $\mathrm{H} 1$ with forced selection of CHD death as the endpoint but flexibility in the selection of the analysis set and covariate specification (24 configurations), Strategy A was unbiased with $E[\hat{\theta}]=-0.1620$ being very close to the relevant estimand of $\theta=\ln (0.85)=$ -0.1625 (Fig. 3a). The minimum and maximum $E[\hat{\theta}]$ value from the subset of analysis configurations that included one or more covariates was -0.1627 and -0.1610 , respectively. Thus, whilst inclusion of prognostic covariates in a Cox proportional hazards regression model technically changes the measure of the treatment effect from a marginal measure to a conditional measure, the $E[\hat{\theta}]$ values from the analysis configurations that included covariates were good estimates of $\theta$. Under Strategy B2, the density of $\hat{\theta}$ was shifted to the left with $E[\hat{\theta}]=-0.1944$, reflecting a relative bias of $20 \%$.

In the second series run under $\mathrm{H} 1$ with no constraint being imposed on analysis configuration (72 configurations), Strategy A was unbiased with $E[\hat{\theta}]=-0.1232$ being close to the relevant estimand $\theta=-0.1227$ (Fig. 3b). Under Strategy B, however, $E[\hat{\theta}]=-0.1980$ reflected a relative bias of $61 \%$. (a)

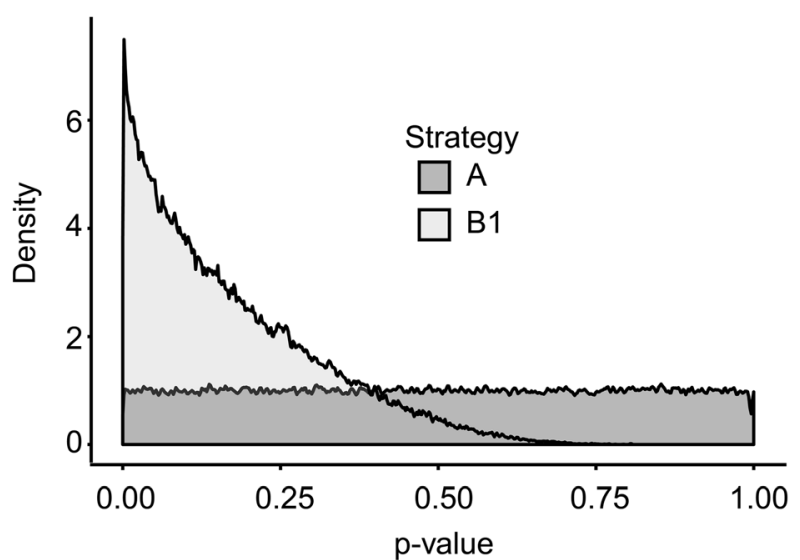

(b)

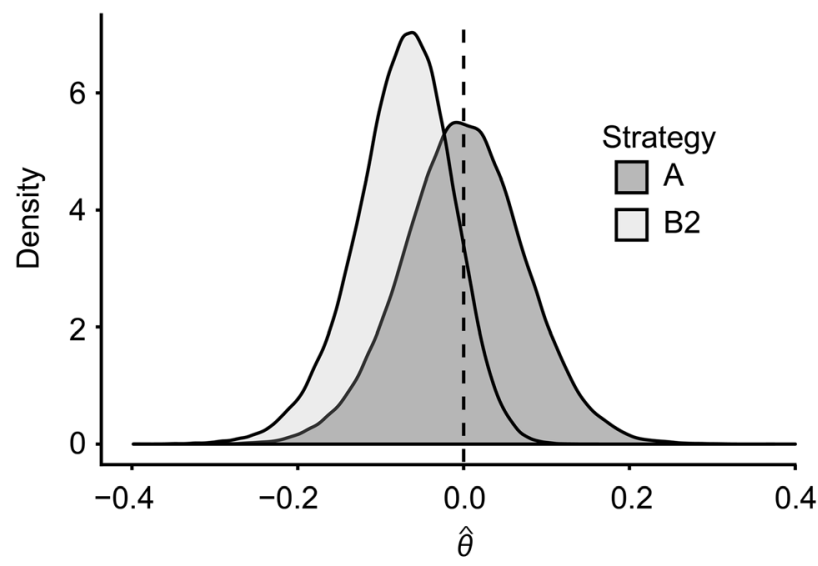

Fig. 1 Distribution of $p$-values and $\hat{\theta}$ under $\mathrm{HO}$ (null hypothesis) (Scenario 1) 


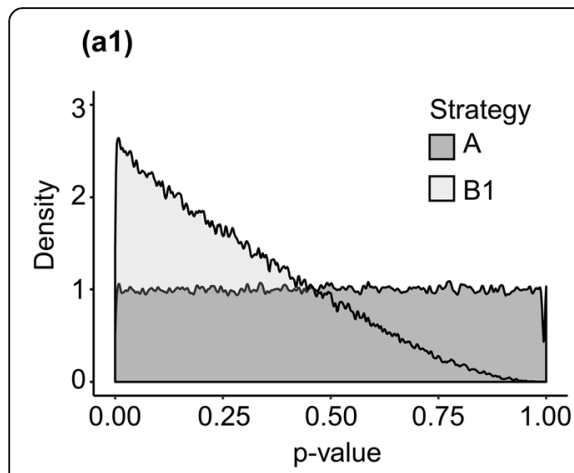

(a2)

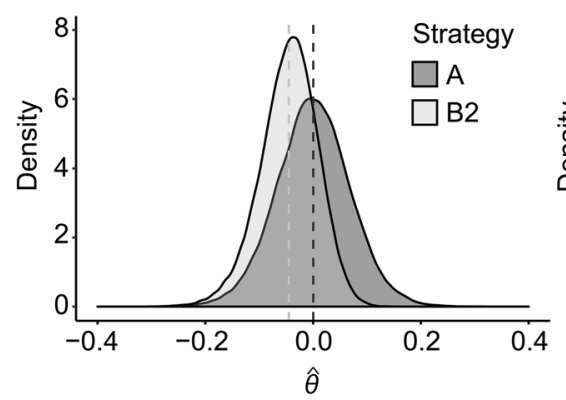

(b1)

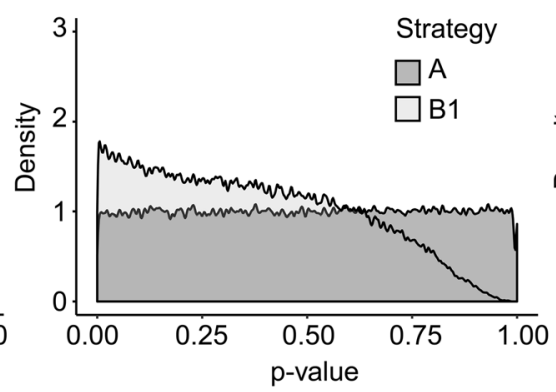

(b2)

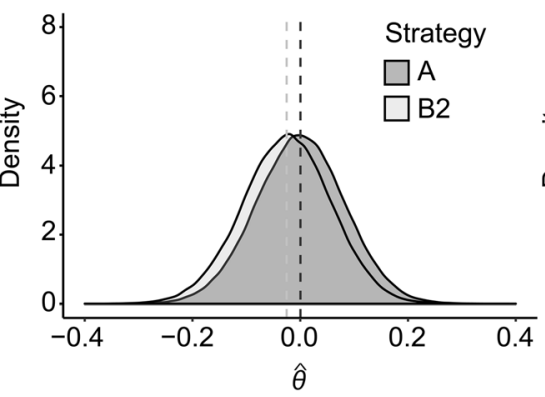

(c1)

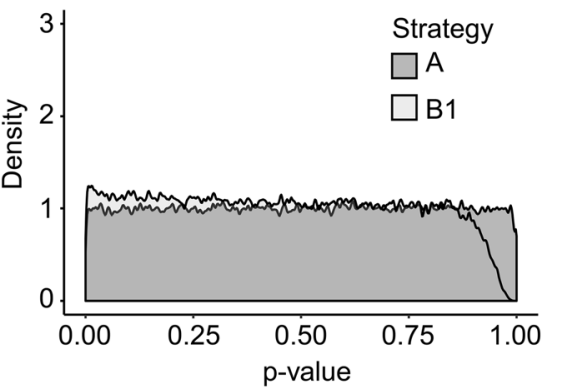

(c2)

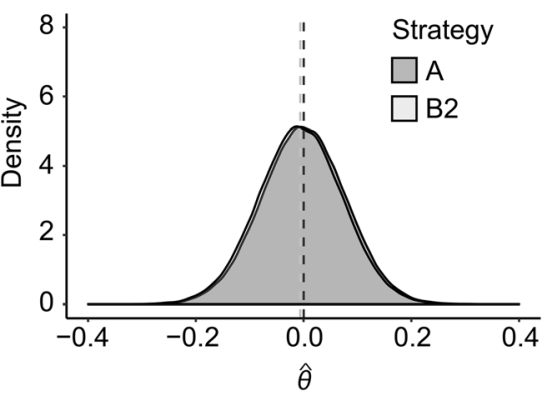

Fig. 2 Distribution of $p$-values and $\hat{\theta}$ under $\mathrm{HO}$ (null hypothesis) and flexibility in only one analysis decision category (Scenario 2)

\section{All analysis configurations}

The distribution of $\hat{\theta}$ across all 72 possible analysis configurations under $\mathrm{H} 0$ and $\mathrm{H} 1$ is presented as a series of boxplots in Additional file 2.

\section{Discussion}

This study demonstrated how estimates of treatment effect (under $\mathrm{HO}$ and $\mathrm{H} 1$ ) can be sensitive to the approach used to inform decisions to change aspects of an ongoing RCT. The legitimate decision strategy (Strategy A) produced results that were valid (consistent with expectations) under both $\mathrm{HO}$ and $\mathrm{H} 1$. This is because all 72 analysis configurations produced $E[\hat{\theta}]$ values that were consistent with expectations (see Additional file 2). Thus, whilst our decision to specify a 50\% switching probability under Strategy A was (a)

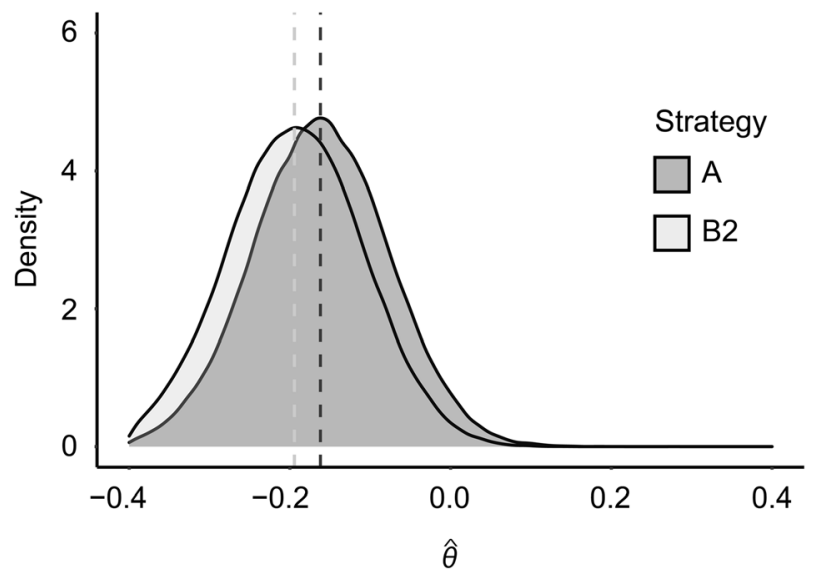

(b)

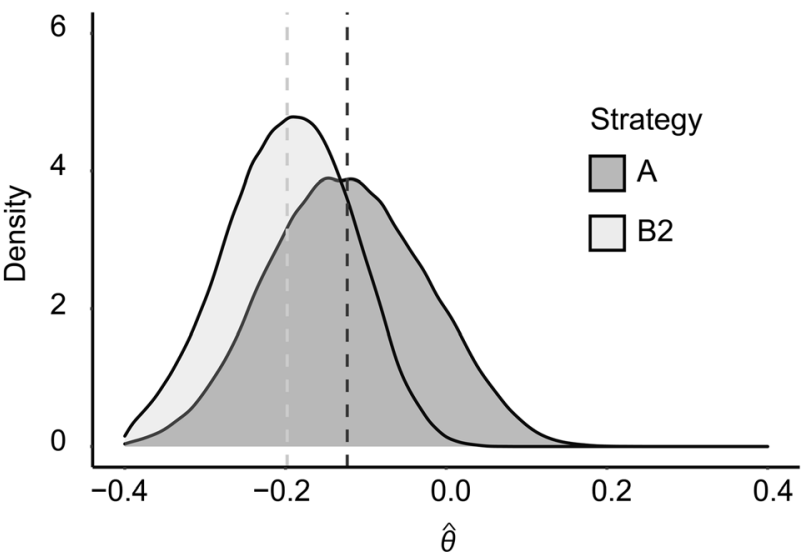

Fig. 3 Distribution of $\hat{\theta}$ under $\mathrm{H} 1$ (alternative hypothesis) (Scenario 3) 
arbitrary, the switching probability specified does not change the type 1 error probability or $E[\hat{\theta}]$ under H0. For example, setting the Strategy A switching probability to $100 \%$ under HO produces a type 1 error rate of 0.0504 (95\% CI: 0.0491 to 0.0518 ) and $E[\hat{\theta}]=0.000$.

The type 1 error rate of the illegitimate decision strategies (Strategies B1 and B2) was well in excess of the 5\% nominal significance level (reaching 23\% with Strategy B1; Fig. 1), and $E[\hat{\theta}]$ was substantially biased (e.g. a relative bias of $61 \%$ was observed with Strategy B2 under H1; Fig. 3).

The three individual analysis decision categories did not contribute equally to the bias under $\mathrm{HO}$ for Strategies B1 and B2. We observed the largest bias being attributable to changing the endpoint, followed by changing the analysis set composition and, finally, by changing the covariate specification. This pattern is not generalisable, however, as it is a product of the unique joint distribution of outcomes and covariates in the LIPID dataset. The result does, however, demonstrate the potential for substantial bias even if flexibility is restricted to only a few configurations.

The set of scenarios and analysis configurations investigated was far from exhaustive. We did not, for example, explore the consequences of switching between an analysis that accounts for 'centre' effects and one that does not, even though the LIPID trial was a multicentre trial. Our results nevertheless illustrate the fact that otherwise methodologically appropriate reactive revisions and/or discretionary decisions can be made without introducing a bias, provided they are made completely independently of knowledge of the association between treatment assignment and outcome. This is a more straightforward proposition in cases where the impetus for a change to a double-blinded RCT is based on information external to the trial, but may be more challenging to follow for open-label RCTs and/or when based on information from within the RCT. Our simulation study did not examine the effect of changes that are driven by within-trial factors (e.g. Example 1 in Table 1). These will be the subject of future simulation studies undertaken by our group.

A compelling rationale for changing some aspect of the planned design/analysis of an RCT is potentially more likely to arise in cases where there have been few preliminary data to guide the original plan, and/or when the RCT takes several years to undertake (and new evidence comes to light and/or the clinical landscape changes). Seamless phase II/III trial designs are a good strategy for accommodating the former situation. These include designs that allow for new treatment arms to be added and other (ineffective) treatment arms to be removed, and for decisions to be based on a synthesis of intermediate and definitive endpoint data. Such designs involve statistical concepts that are likely to be less well understood by clinicians undertaking RCTs, and may be challenging to fund via conventional grant schemes for investigator-initiated research (where they will compete for funding with other research proposals with simper statistical designs and more straightforward implementation plans). Traditional RCTs are thus likely to remain a popular design for investigator-initiated research.

Trialists undertaking traditional RCTs should be conscious of the potential for new information to provoke a need to change some aspect of their ongoing trial, and the potential risks to the integrity of the trial if this is not managed appropriately. Appropriate management will involve carefully documenting the rationale and process for making changes to ensure reviewers/readers can make appropriate assessments of the legitimacy of the changes [19].

The SPIRIT statement provides general guidance on what should be included in clinical trial protocols, but it does not provide detailed instruction on the process of documenting changes in protocol amendments once the trial has started [20]. The CONSORT statement provides general guidance on the reporting of changes to the trial outcomes and methods; however, reviews suggest there remains room for improvement $[14,21-$ 23]. A supplement to the current guidelines and checklists would be useful. Ongoing initiatives to promote greater detail in pre-specified endpoint definitions also merit support [23-26].

Gaining access to full trial protocols remains a challenge. Comparisons of planned versus reported clinical trial procedures, methods and analyses have largely involved the review of limited publicly available information from clinical registries $[9,13,27,28]$. Such limited information is potentially insufficient, given evidence that access to more detailed (not publicly available) information can change reviewers' opinions [29-34].

Improved access to full protocols, e.g. via existing clinical trial registries, would facilitate the fair appraisal of trial results and deter selective reporting [29, 35]. Counterarguments to such transparency, e.g. that hinge on the need to maintain confidentiality of (commercially sensitive) intellectual property and/or information integral to preserving treatment concealment [29, 36-38], have been challenged by regulatory authorities and others [29, 31, 34, 39]. Even protocols with sensitive information could be prospectively lodged, and the information kept confidential in escrow until study results are disclosed [37]. The implementation of research assessment indicators by academic research institutions, which publicly laud trialists who share their protocols, and journals enforcing policies and building capacity for public sharing, including mandating access to full and 
complete protocols as opposed to abridged versions, are also pragmatic steps to change.

\section{Conclusions}

This study demonstrated how modifications to the planned design/analysis of an RCT can be made validly, and illustrated how illegitimate changes introduce bias. For example, using illegitimate approaches, we obtained a type 1 error rate under $\mathrm{H} 0$ of $23 \%$, and a relative bias of $61 \%$ under H1. Studies in other settings have highlighted how flexibility in the analysis approach can inflate the type 1 error [7, 40, 41], but none have focused on the implications of changing the planned design/analysis of RCTs. Our findings thus provide important guidance for trialists (and stakeholders) on how to make a methodologically justifiable change to a planned RCT design/analysis, and highlight the circumstances under which a change can lead to bias. In so doing, our research intends to both encourage trialists to consider how changes are made and documented, and allow clinicians and stakeholders to distinguish between changes that could lead to a systematic bias versus those that do not. This is important for ensuring that methodically unsound changes to RCTs are recognised and avoided, and that RCTs which have undergone methodically sound changes are not incorrectly dismissed as potentially biased.

\section{Supplementary information}

Supplementary information accompanies this paper at https://doi.org/10. 1186/s13063-020-04374-3.

Additional file 1. Construction of the simulated trial datasets and analysis configurations

Additional file 2. Boxplots of $\theta^{\wedge}$ for all 72 analysis configurations under $\mathrm{HO}$ and $\mathrm{H} 1$.

\section{Abbreviations}

AMl: Acute myocardial infarction; CHD: Coronary heart disease: CONSORT: Consolidated Standards of Reporting Trials; HO: Null hypothesis; H1: Alternative hypothesis; HR: Hazard ratio; MAMS: Multi-arm multi-stage; RCT: Randomised controlled trial; SPIRIT: Standard Protocol Items:

Recommendations for Interventional Trials; UAP: Unstable angina pectoris

\section{Acknowledgements}

The authors would like to thank the LIPID Trial Management Committee for granting the authors permission to access a copy of the LIPID dataset.

\section{Authors' contributions}

JS and AJM conceived and designed the experiments. AJM MS and XC performed the experiments. All authors analysed and interpreted the data and wrote, read and approved the final manuscript.

\section{Funding}

This work was supported by the National Health and Medical Research Council (NHMRC) (Postgraduate scholarship number APP1168666) in addition to a program grant from the NHMRC. The NHMRC had no involvement in the collection, analysis and interpretation of data; in the writing of the report; and in the decision to submit the article for publication.

\section{Availability of data and materials}

The data that support the findings of this simulation study are available from the LIPID Trial Management Committee; however, restrictions may apply to the availability of these data, due to relevant data protection laws.

Ethics approval and consent to participate

Consent to participate is not applicable. Ethics approval was granted by the University of Sydney Ethics Committee (Study Approval ID: 2019/579).

\section{Consent for publication}

Not applicable.

\section{Competing interests}

The authors declare that they have no competing interests.

\section{Author details}

${ }^{1}$ The National Health and Medical Research Council Clinical Trial Centre, University of Sydney, Camperdown, NSW 2050, Australia. ${ }^{2}$ Department of Mathematics and Statistics, Macquarie University, Macquarie Park, NSW, Australia. ${ }^{3}$ Janssen-Cilag Pty. Limited, Macquarie Park, NSW, Australia.

Received: 23 December 2019 Accepted: 5 May 2020

Published online: 03 June 2020

\section{References}

1. Sydes MR, et al. Issues in applying multi-arm multi-stage methodology to a clinical trial in prostate cancer: the MRC STAMPEDE trial. Trials. 2009;10(1):39.

2. Berry DA. Bayesian clinical trials. Nat Rev Drug Discov. 2006;5(1):27.

3. FIELD Study Investigators. Effects of long-term fenofibrate therapy on cardiovascular events in 9795 people with type 2 diabetes mellitus (the FIELD study): randomised controlled trial. Lancet. 2005;366(9500):1849-61.

4. Jonker DJ, et al. Cetuximab for the treatment of colorectal cancer. N Engl J Med. 2007:357(20):2040-8.

5. Karapetis CS, et al. K-ras mutations and benefit from cetuximab in advanced colorectal cancer. N Engl J Med. 2008:359(17):1757-65.

6. Gill G, SNAC Trial Group of the Royal Australasian College of Surgeons (RACS) and NHMRC Clinical Trials Centre. Sentinel-lymph-node-based management or routine axillary clearance? One-year outcomes of sentinel node biopsy versus axillary clearance (SNAC): a randomized controlled surgical trial. Ann Surg Oncol. 2009;16(2):266-75.

7. Kahan $B C$, Jairath $\vee$. Outcome pre-specification requires sufficient detail to guard against outcome switching in clinical trials: a case study. Trials. 2018; 19(1):265.

8. Fleming PS, et al. Outcome discrepancies and selective reporting: impacting the leading journals? PLoS One. 2015;10(5):e0127495.

9. Dwan $\mathrm{K}$, et al. Comparison of protocols and registry entries to published reports for randomised controlled trials. Cochrane Database Syst Rev. 2011; $1: 1-51$

10. Dwan $\mathrm{K}$, et al. Evidence for the selective reporting of analyses and discrepancies in clinical trials: a systematic review of cohort studies of clinical trials. PLoS Med. 2014;11(6):e1001666.

11. Page MJ, et al. Bias due to selective inclusion and reporting of outcomes and analyses in systematic reviews of randomised trials of healthcare interventions. Cochrane Database Syst Rev. 2014;(10). https://doi.org/10. 1002/14651858.MR000035.pub2.

12. Altman DG, Moher D, Schulz KF. Harms of outcome switching in reports of randomised trials: CONSORT perspective. BMJ. 2017;356:3396.

13. Jones CW, et al. Comparison of registered and published outcomes in randomized controlled trials: a systematic review. BMC Med. 2015:13:282.

14. Turner $L$, et al. Consolidated Standards of Reporting Trials (CONSORT) and the completeness of reporting of randomised controlled trials (RCTs) published in medical journals. Cochrane Database Syst Rev. 2012;11: MR000030.

15. Krauss A. Why all randomised controlled trials produce biased results. Ann Med. 2018;50(4):312-22.

16. Goldacre, B. Where does Annals of Internal Medicine stand on outcome switching? A detailed response. 2016 Retrieved 26 May 2018; Available from: (http://compare-trials.org/blog/where-does-annals-of-internalmedicine-stand-on-outcome-switching-a-detailed-response/).

17. Morris TP, White IR, Crowther MJ. Using simulation studies to evaluate statistical methods. Stat Med. 2019;38(11):2074-102. 
18. LIPID Study Group. Prevention of cardiovascular events and death with pravastatin in patients with coronary heart disease and a broad range of initial cholesterol levels. N Engl J Med. 1998;339(19):1349-57.

19. Ioannidis JP, Caplan AL, Dal-Ré R. Outcome reporting bias in clinical trials: why monitoring matters. BMJ. 2017;356:1408.

20. Chan A, et al. Spirit 2013 Statement: Defining standard protocol items for clinical trials. Ann Intern Med. 2013;158(3):200-7.

21. Ebrahim S, et al. Reanalyses of randomized clinical trial data. JAMA. 2014; 312(10):1024-32.

22. Naudet $F$, et al. Data sharing and reanalysis of randomized controlled trials in leading biomedical journals with a full data sharing policy: survey of studies published in The BMJ and PLOS Medicine. BMJ. 2018;360:k400.

23. Butcher NJ, et al. Improving outcome reporting in clinical trial reports and protocols: study protocol for the Instrument for reporting Planned Endpoints in Clinical Trials (InsPECT). Trials. 2019:20(1):161.

24. COMET. COMET: Core Outcome Measures in Effectiveness Trials. Available from: http://www.comet-initiative.org/. Accessed 11 Dec 2019.

25. COSMIN. COSMIN: COnsensus based Standards for the selection of health Measurement INstruments. Available from: https://www.cosmin.nl/. Accessed 11 Dec 2019

26. InsPECT. InsPECT: Instrument for reporting Planned Endpoints in Clinical Trials. Available from: https://www.inspect-statement.org/. Accessed 11 Dec 2019.

27. Chen T, et al. Comparison of clinical trial changes in primary outcome and reported intervention effect size between trial registration and publication. JAMA Netw Open. 2019;2(7):e197242.

28. Jones $\mathrm{CW}$, et al. Primary outcome switching among drug trials with and without principal investigator financial ties to industry: a cross-sectional study. BMJ Open. 2018;8(2):e019831.

29. Chan A-W, Hróbjartsson A. Promoting public access to clinical trial protocols: challenges and recommendations. Trials. 2018;19(1):116.

30. Eichler $\mathrm{H}-\mathrm{G}$, et al. Open clinical trial data for all? A view from regulators. PLoS Med. 2012;9(4):e1001202.

31. European Medicines Agency policy on publication of clinical data for medicinal products for human use, POLICY/0070, EMA/144064/2019. 2019. https://www.ema.europa.eu/en/documents/other/european-medicinesagency-policy-publication-clinical-data-medicinal-products-human-use_en. pdf.

32. Mayo-Wilson E, et al. Cherry-picking by trialists and meta-analysts can drive conclusions about intervention efficacy. J Clin Epidemiol. 2017;91:95-110.

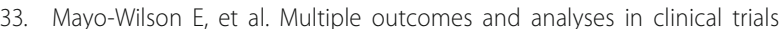
create challenges for interpretation and research synthesis. J Clin Epidemiol. 2017;86:39-50.

34. Doshi P, Jefferson T, Del Mar C. The imperative to share clinical study reports: recommendations from the Tamiflu experience. PLoS Med. 2012; 9(4):e1001201.

35. Schmucker C, et al. Extent of non-publication in cohorts of studies approved by research ethics committees or included in trial registries. PLoS One. 2014;9(12):e114023.

36. Ross $\mathrm{S}$, et al. Protecting intellectual property associated with Canadian academic clinical trials_approaches and impact. Trials. 2012;13:243.

37. Basu AP, Pearse JE, Rapley T. Publishing protocols for trials of complex interventions before trial completion-potential pitfalls, solutions and the need for public debate. Trials. 2017;18(1):5.

38. Reveiz L, et al. Do trialists endorse clinical trial registration? Survey of a PubMed sample. Trials. 2007:8(1):30

39. Mayo-Wilson E, Doshi P, Dickersin K. Are manufacturers sharing data as promised? BMJ. 2015;351:h4169.

40. Simmons JP, Nelson LD, Simonsohn U. False-positive psychology: undisclosed flexibility in data collection and analysis allows presenting anything as significant. Psychol Sci. 2011:22(11):1359-66.

41. Counsell CE, et al. The miracle of DICE therapy for acute stroke: fact or fictional product of subgroup analysis? BMJ. 1994;309(6970):1677-81

\section{Publisher's Note}

Springer Nature remains neutral with regard to jurisdictional claims in published maps and institutional affiliations.

\section{Ready to submit your research? Choose BMC and benefit from:}

- fast, convenient online submission

- thorough peer review by experienced researchers in your field

- rapid publication on acceptance

- support for research data, including large and complex data types

- gold Open Access which fosters wider collaboration and increased citations

- maximum visibility for your research: over $100 \mathrm{M}$ website views per year

At BMC, research is always in progress.

Learn more biomedcentral.com/submissions 